\title{
Diagnostische Verfahren, klinische Merkmale und Beratung bei Mukoviszidose (cystischer Fibrose, CF)
}

\author{
Kris De Boeck \\ Department of Pediatrics, University of Leuven, Leuven, Belgium
}

\begin{abstract}
Schlüsselwörter
Cystische Fibrose - Schweisstest · CFTR-Mutation - Messung der nasalen Potenzialdifferenz • Salzverlust-Syndrom •

Pseudomonas aeruginosa $\cdot$ Pankreasinsuffizienz $\cdot$ Infertilität
\end{abstract}

\section{Zusammenfassung}

Bei den meisten Patienten, bei denen schliesslich eine Mukoviszidose (cystische Fibrose, CF) diagnostiziert wird, treten in den ersten Lebensmonaten klassische Symptome auf, wie z.B. Mekonium-lleus, Gedeihstörungen sowie rezidivierender oder persistierender Husten. Die Krankheitssymptome treten offen zutage, sind progressiv und betreffen diverse Organsysteme. Standardtest bei der Diagnose dieser Erkrankung ist der Schweisstest. Eine Chloridkonzentration von $>60 \mathrm{mmol} / \mathrm{l}$ bestätigt den klinischen Verdacht und sichert die Diagnose. Seit Entdeckung der Mutationen des CFTR (Cystische Fibrose Transmembran Regulator)-Gens ist mittlerweile klar geworden, dass das klinische Spektrum der Erkrankung breit gefächert ist. Es wurde bereits der Nachweis erbracht, dass mehrere Patientengruppen zwei CFTRMutationen in sich tragen, und zwar Patienten mit klassischer $\mathrm{CF}$, Patienten mit weniger stark ausgeprägten Symptomen, bei denen die Erkrankung erst in der Jugend oder sogar erst im Erwachsenenalter auftritt, und Patienten mit nur einem klinischen Merkmal, z.B. rezidivierender Pankreatitis, sklerosierender Cholangitis, 'idiopathischer' Bronchiektasie und Infertilität. Bei den beiden letzten Kategorien spricht man von einer atypischen CF. Diese Patienten müssen wissen, dass sie nicht unter einer typischen CF leiden, aber dass sie dennoch ein gewisses Risiko für eine der Mukoviszidose ähnliche Erkrankung haben und daher von Zeit zu Zeit von Ärzten, die mit dem äusserst breitgefächerten Spektrum dieser Erkrankung vertraut sind, untersucht und überwacht werden müssen. Die am häufigsten bei Patienten mit typischer CF auftretenden Symptome sind chronisch-progressive, bakterielle Infekte und Entzündungen der oberen und unteren Atemwege, die sich zeitweilig verschlechtern. Bei 90\% der Patienten liegt eine Insuffizienz der Bauchspeicheldrüse vor. Die betroffenen Männer sind nach der Pubertät zeugungsunfähig. Bei Patienten mit Mukoviszidose können Symptome und Komplikationen in den Nebenhöhlen, in der Lunge, in der Bauchspeicheldrüse, in der Leber, im Darm, im Ösophagus, in den Knochen und in den Gelenken etc. auftreten. Die komplexe Mischung möglicher Krankheitssymptome bedeutet, dass jeder Patient einzigartig ist. Mit zunehmendem Alter nimmt die Zahl der Komplikationen tendenziell zu und die körperliche Verfassung verschlechtert sich. Aufgrund der fortgeschrittenen Lungenerkrankung können sich viele Mukoviszidose-Patienten im jungen Erwachsenenalter nicht mehr sportlich betätigen und $20 \%$ leiden unter einem mit der Mukoviszidose in Zusammenhang stehenden Diabetes mellitus. Bei der typischen CF handelt es sich um eine lebensverkürzende Erkrankung und die mittlere Überlebensdauer beträgt derzeit selbst bei intensivster Behandlung und Beobachtung nur ungefähr 34 Jahre. Auf den Patienten und ihren Familien lastet daher ein enormer psychischer Druck. Beratung ist somit von äusserster Wichtigkeit und sehr komplex.

Copyright ๑ 2006 Nestec Ltd., Vevey/S. Karger AG, Basel

\section{KARGER}

Fax +4161306 1234

E-Mail karger@karger.ch

www.karger.com (c) 2006 Nestec Ltd., Vevey/S. Karger AG, Basel

0250-9652/06/0643-0121\$23.50/0

Online-Zugriff auf:

www.karger.com/and
Prof. Dr. K. De Boeck

Department of Pediatrics

Herestraat 49, BE-3000 Leuven (Belgium)

Tel. +32 16343820 , Fax +32 16343842

E-Mailchristiane.deboeck@uz.kuleuven.ac.be 


\section{Einleitung}

Die Mukoviszidose (cystische Fibrose, CF) ist bei Menschen mit weisser Hautfarbe die am häufigsten vorkommende lebensverkürzende Erbkrankheit. Bei Neugeborenen schwankt die Inzidenz stark je nach Ethnizität der untersuchten Population. Seit Entdeckung des CFTR (Cystische Fibrose Transmembran Regulator)-Gens ist klar, dass die CF in allen bisher untersuchten Populationen auftritt und ihre Inzidenz bei Menschen anderer Hautfarben bisher tendenziell unterschätzt wurde [1].

Die CF ist eine autosomal rezessive Erbkrankheit, die durch eine Mutation in einem der Gene verursacht wird, die für das CFTR-Protein kodieren. Bei der CF handelt es sich um eine Multiorganerkrankung. Die Symptome der Erkrankung entstehen durch den mangelhaften Chloridtransport über das Epithel der exokrinen Drüsen, der durch das Fehlen oder die Störung des cAMP-aktivierten CFTR-Chloridkanals verursacht wird [2].

Bei den mit Abstand meisten Patienten manifestiert sich die Erkrankung bei Geburt oder in den ersten Lebensmonaten, wobei die Diagnostizierung der Erkrankung kein Problem darstellt, solange die dazu erforderlichen Untersuchungen sorgfältig sind und ein Schweisstest bei einem erfahrenen Labor durchgeführt wird. Bei einigen Patienten ist die Erkrankung jedoch weniger ausgeprägt und dann äusserst schwer zu diagnostizieren. Bei diesen Patienten äussert sich die Krankheit möglicherweise durch unterschiedliche Symptome und der Schweisstest ergibt häufig kein schlüssiges Ergebnis. Weitere diagnostische Tests können bei der Feststellung der endgültigen Diagnose behilflich sein, u.a. die Suche nach zwei krankheitsursächlichen CFTR-Mutationen oder der physiologische Nachweis einer CFTR-Proteinstörung [3].

\section{Symptome, die auf eine zystischen Fibrose hinweisen}

Da die cystische Fibrose bei Menschen mit weisser Hautfarbe die gefährlichste Erbkrankheit ist, sollte sie im Rahmen der Differenzialdiagnose bei Säuglingen mit den verschiedensten rezidivierenden oder persistierenden Symptomen häufig in Betracht gezogen werden. In der Tat kann es sich bei vielen Symptomen um die ersten Anzeichen einer cystischen Fibrose handeln.

Zehn bis 15\% der Kinder mit cystischer Fibrose leiden nach der Geburt unter Erbrechen und Ausbleiben des Stuhlgangs [4]. Mekonium-Ileus tritt bei CF so häufig auf, dass die Diagnose fast nie übersehen wird. Ein weniger bekanntes Symptom im Neugeborenenalter ist die anhaltende cholestatische Gelbsucht [5]. Häufigstes Symptom der CF sind die aufgrund der exokrinen Insuffizienz der Bauchspeicheldrüse auftretenden Gedeihstörungen, die oft mit einem chronischen Husten einhergehen [6]. Andererseits kann die Verdauung bei Kindern mit CF monate- oder sogar jahrelang vollkommen normal sein [7].

Bereits im Säuglings- bzw. Kleinkindalter können rezidivierender oder persistierender Husten sowie rezidivierende oder chronische Infekte der unteren Atemwege einsetzen. Chronischer Husten wird häufig durch eine Virusinfektion, z.B. eine RSV (respiratory syncytial virus)-Infektion ausgelöst [8].

Salzverlustsyndrome treten hauptsächlich in wärmeren Klimaregionen auf. Ein mit Dehydratation und Elektrolytungleichgewicht aufgrund übermässiger Salzverluste einhergehender Hitzschlag kann ein Hinweis auf das Vorliegen einer CF sein [9]. Aber auch in gemässigterem Klima leiden Säuglinge und Kleinkinder mit CF möglicherweise unter Apathie, schlechter Nahrungsaufnahme, Erbrechen, einer geringen Gewichtszunahme und einer Polyurie (Bartter-like Syndrome) aufgrund hypochlorämischer Alkalose und Hypokalämie nach unzureichender Salzaufnahme [9].

Wird die Diagnose im Kleinkindalter übersehen, dann können im weiteren Krankheitsverlauf Komplikationen eintreten, die letztendlich zur Diagnose CF führen, u.a. Trommelschlegelfinger, Bronchiektasen, chronische Sinusitis, Nasenpolypen, Leberzirrhose, Hämatemesis, Hämoptyse, etc. Die Liste der Symptome, die auf das Vorliegen einer CF hinweisen, ist lang (Tabelle 1). Der unerfüllte Kinderwunsch bei einem Paar, der dazu führt, dass beim Mann eine Azoospermie aufgrund des Fehlens beider Samenleiter festgestellt wird, ist das klassische Beispiel für einen atypischen CF-Phänotyp [10; Cutting, S. 113].

Bei der Frage nach der Ursache von Gedeihstörungen in Verbindung mit rezidivierenden Infekten der unteren Atemwege bei einem Säugling oder Kleinkind wird jeder Arzt sofort die Diagnose CF als Möglichkeit vorbringen. Trotzdem wird die Diagnose selbst bei Patienten mit klassischen Symptomen regelmässig übersehen oder erst mit Verzögerung erkannt. Die Gründe hierfür können vielfältig sein. Möglicherweise formulieren die Eltern die Hauptbeschwerden ungenau und sagen nicht: 'Mein Kind isst viel, hat fetten Stuhl und nimmt nicht an Gewicht zu', sondern: 'Mein Kind ist gereizt und schwer zu beruhigen'. Möglicherweise wechseln die Eltern den Arzt, anstatt bei einem ungelösten Problem nochmals den gleichen Arzt zu 
Tabelle 1. Klinische Symptome, die auf CF hinweisen

\begin{tabular}{ll}
\hline Starker Hinweis & Weniger konkreter Hinweis \\
\hline $\begin{array}{l}\text { Gastrointestinale Symptome } \\
\text { Mekonium-Ileus }\end{array}$ & Gedeihstörungen \\
Exokrine Bauchspeicheldrüseninsuffizienz & Hypoproteinämie \\
bei Kindern & Mangel an fettlöslichen Vitaminen \\
& Distales intestinales Obstruktionssyndrom (DIOS) \\
& Rektaler Prolaps \\
& Biliäre Zirrhose \\
& Portale Hypertonie \\
& Gallensteine bei Kindern ohne hämolytische Erkrankung \\
& Primär sklerosierende Cholangitis \\
& Exokrine Bauchspeicheldrüseninsuffizienz bei \\
& Erwachsenen \\
& Rezidivierende Pankreatitis \\
& \\
\hline
\end{tabular}

Sinopulmonale Symptome

Persistierender Atemwegsinfekt mit Pseudomonas aeruginosa im Lungensekret Bronchiektasen in beiden oberen Lungenlappen Persistierender Atemwegsinfekt mit Burkholderia cepacia

Nasenpolypen bei Kindern
Persistierende oder rezidivierende Atemwegsinfekte mit Staphylococcus aureus, P. aeruginosa, Achromobacter xylosoxidans oder Haemophilus influenzae

Radiologischer Nachweis von Bronchiektasen, Atelektasen, Hyperinflation oder persistierender Infiltrate in Röntgenaufnahmen des Brustraums Hämoptyse in Verbindung mit einer diffusen pulmonalen Erkrankung ausser Tuberkulose oder Vaskulitis Chronischer bzw. produktiver Husten Allergische bronchopulmonale Aspergillose

Nasenpolypen bei Erwachsenen

Röntgennachweis einer chronischen Pansinusitis

Sonstige

Hypochlorämische Alkalose ohne Erbrechen Angeborenes Fehlen der Samenleiter auf beiden Seiten
Trommelschlegelfinger

Osteopenie/Osteoporose im Alter von unter 40 Jahren Atypischer Diabetes

Aus De Boeck et al. [55], mit freundlicher Genehmigung der BMJ Publishing Group.

konsultieren. Möglicherweise übersieht man das Gesamtbild und geht nur einem der durch die Malabsorption verursachten sekundären Probleme nach, z.B. Ödemen und Anämie mit Überweisung des Kindes an einen Nierenfacharzt; Überblähung der Lunge, Lebervergrösserung und Ernährungsschwierigkeiten mit Überweisung an einen pädiatrischen Kardiologen; Hämatome mit Überweisung an einen Kinder-Onkologen; Ausschläge perianal und perioral mit Überweisung an einen Dermatologen sowie Anämie und Hämolyse mit Überweisung an einen pädiatrischen Hämatologen. Alle oben genannten Fälle wurden bereits in Berichten behandelt [11]. Ferner sind die Kinder häufig nicht akut krank. Der mit dem chronischen
Atemwegsinfekt einhergehende Husten ist anfangs nicht allzu stark ausgeprägt, sondern eher sehr hartnäckig. Die respiratorischen Probleme nehmen langsam, aber stetig, zu. Viele Kinder kommen mit rezidivierenden Infekten der unteren Atemwege zu einem Allgemeinarzt, der die Diagnose CF möglicherweise verwirft, weil das Kind zu gesund aussieht bzw. weil keine Gedeihstörungen vorliegen. Bei Kindern mit chronischen Beschwerden erfordert die Diagnose einen hohen Verdachtsindex. Ebenso sollten sicherlich alle Kinder, die in der Lage sind, Sputum zu produzieren, die unter rezidivierenden Infiltraten der unteren Atemwege leiden, bei denen sich der Husten unter Antibiotikabehandlung nur vorübergehend bessert, die im Som- 
mer nicht hustenfrei sind, bei denen der Erfolg einer Asthmabehandlung ausbleibt bzw. alle Kinder mit Trommelschlegelfingern mittels eines Schweisstest auf Mukoviszidose untersucht werden.

Eine frühzeitige Diagnose ist äusserst wichtig, um die Progression der Krankheit hinauszuzögern. Aus diesem Grund ist ein Neugeborenen-Screening in den Ländern, die die Kosten tragen können, bzw. insbesondere in Regionen, in denen das Durchschnittsalter bei der Diagnose hoch ist, empfehlenswert [12]. Ein NeugeborenenScreening ist jedoch nicht ganz unproblematisch, da eine solche Untersuchung auch zu falsch negativen und falsch positiven Testergebnissen (heterozygote aber klinisch unauffällige Merkmalsträger) führt [13].

\section{Spezifische Verfahren für die CF-Diagnose}

Die folgenden Verfahren eignen sich zur Absicherung der CF-Diagnose: der Schweisstest, die DNA-Analyse von CFTR-Mutationen und Bioassays zum Nachweis von CFTR-Mutationen. Ist der sichere Gibson und Cooke [14] Schweisstest verfügbar, so eignet sich dieser aufgrund seiner hervorragenden diagnostischen Ausbeute (der Test ist bei $98 \%$ der amerikanischen CF-Patienten positiv) am besten zur Erstdiagnose [15]. Bei einer 'First-Line' DNA-Analyse bezüglich CFTR-Mutationen wird der Patient auf die in seiner Population am häufigsten auftretenden CF-ursächlichen CFTR-Mutationen untersucht, d.h. auf CFTR-Mutationen mit einer Prävalenz von $>0,5 \%$. In den meisten Populationen ergibt eine solche Untersuchung bei 80-95\% der Patienten zwei CFTR-Mutationen [1]. Bei Patienten mit atypischen Krankheitssymptomen ergibt der Schweisstest häufig kein eindeutiges Ergebnis. In diesen Fällen sind weitere diagnostische Untersuchungen, d.h. eine CFTR-Mutationsanalyse und manchmal CFTR-Bioassays, zur Absicherung der Diagnose erforderlich.

\section{Schweisstest}

Bei einem korrekt ausgeführten Schweisstest ist die diagnostische Ausbeute hoch. Dieser 'einfache' Test erfordert jedoch grosse Fachkenntnis und peinlichste Detailgenauigkeit. In vielen Laboratorien werden $\mathrm{zu}$ wenige Tests von $\mathrm{zu}$ vielen verschiedenen Labortechnikern durchgeführt, so dass die Fehlerwahrscheinlichkeit hoch ist [16]. Der Goldstandard ist nach wie vor der 1959 von Gibson und Cooke entwickelte quantitative PilocarpinIontophorese-Schweisstest [14]. Nach Stimulation mit Pilocarpin wird der Schweiss 30 Minuten lang mit einer zuvor gewogenen Gaze oder einem zuvor gewogenen Löschpapier mit niedrigem Natriumchloridgehalt aufgesogen $[3,14,17,18]$. Für den Test benötigt man eine Schweissrate von mindestens $1 \mathrm{~g} / \mathrm{m}^{2}$ Körperoberfläche, eine Schweissmenge von 50-100 mg reicht also aus.

Bei Patienten mit verdächtigen klinischen Symptomen bestätigt eine Chloridkonzentration im Schweiss von $>60$ $\mathrm{mmol} / \mathrm{l}$ die Diagnose CF. Mit Hilfe des Cutoff-Werts von $60 \mathrm{mmol} / \mathrm{l}$ können gesunde Personen zuverlässig von CFPatienten unterschieden werden [19]. Bei einem Chloridwert von $>60 \mathrm{mmol} / \mathrm{l}$ besteht fast immer ein Zusammenhang mit einer CF. Es gibt diesbezüglich nur wenige Ausnahmen: Werte von $>60 \mathrm{mmol} / \mathrm{l}$ wurden bei einigen seltenen Hautkrankheiten und seltenen endokrinen Störungen bzw. Stoffwechselerkrankungen gemeldet, die jedoch leicht von der CF zu unterscheiden sind. Ausserdem beziehen sich diese Werte in erster Linie auf einzelne Fallberichte. Normalerweise beträgt der Chlorid- und Natriumgehalt im Schweiss $<60 \mathrm{mmol} / \mathrm{l}$ bzw. sogar $<30$ $\mathrm{mmol} / \mathrm{l}$ [20]. Bei Kindern, die über $3 \mathrm{~kg}$ wiegen, normal hydriert sind und nicht unter einer signifikanten Krankheit leiden, kann der Schweisstest ab der 3. Lebenswoche durchgeführt werden. Weitere Einzelheiten über den Schweisstest erfahren Sie im Consensus Report [3, 17, 21].

Bei einem Teil der Patienten mit zwei CFTR-Mutationen, d.h. einer Mutation je CFTR-Gen, betragen die Chloridkonzentrationen im Schweiss $<60 \mathrm{mmol} / \mathrm{l}$ [22, 23]. Einen absoluten Cutoff-Wert für die Chloridkonzentration im Schweiss, unterhalb welchem atypische CF-Fälle ausgeschlossen werden können, gibt es in Wirklichkeit nicht [22], aber die meisten Autoren konzentrieren sich bei ihren Untersuchungen auf Patienten, bei denen die Chloridkonzentrationen im Schweiss zwischen 40 und $60 \mathrm{mmol} / \mathrm{l}$ betragen. Eine Studie konzentrierte sich auf Patienten mit Chloridkonzentrationen im Schweiss zwischen 30 und $60 \mathrm{mmol} / \mathrm{l}$ [20], die bei den Patienten mit CF-Symptomen, die sich einem Schweisstest unterzogen, einen Anteil von ungefähr 4\% ausmachten. Bei $23 \%$ dieser Patienten wurden nachträglich zwei CFTR-Mutationen festgestellt [20].

\section{Feststellen von CFTR-Mutationen}

Das CFTR-Gen befindet sich auf dem langen Arm von Chromosom 7 und kodiert für das CFTR-Protein. Dieses Transmembran-Protein wirkt als cAMP-abhängiger Chloridkanal und hat viele Funktionen, u.a. die wichtige Regulierung der Ionen- und Wasserbilanz über das Epithel [2; Donaldson und Boucher, S. 103-109].

Inzwischen wurden über 1500 CFTR-Mutationen identifiziert [24], von denen über 1000 bei Patienten mit 
CF-Symptomen auftreten. Die verbleibenden Mutationen führen entweder zu weniger ausgeprägten Erkrankungen oder verursachen überhaupt keine Krankheit [24]. Bei mehreren Mutationen, d.h. den meisten KlasseI-Mutationen (Nonsense-Mutationen, Splice-Site-Mutationen, out-frame Deletionen/Insertionen) ist der CF-ursächliche Charakter eindeutig. Bei den anderen Mutationen ist nur mittels funktionaler Studien eindeutig festzustellen, ob es sich um CF-ursächliche Mutationen handelt oder nicht. Bisher wurden solche Studien nur bei den häufigsten CFTR-Mutationen durchgeführt. Bei einer seltenen, nach einem kompletten Screening des CFTR-Gens festgestellten Missense-Mutation kann es sich entweder um einen Polymorphismus oder um eine Mutation handeln, die CF verursacht [Cutting, S. 113].

Die CF ist eine autosomal rezessive Erkrankung, d.h. bei CF-Patienten finden sich in beiden CFTR-Genen krankheitsursächliche Mutationen. Ein Patient kann bei identischer CFTR-Mutation auf beiden CFTR-Allelen homozygot oder bei zwei verschiedenen CFTR-Mutationen compound-heterozygot sein.

Die Häufigkeit der CFTR-Mutationen unterscheidet sich je nach Population [1]. Die bei weitem am häufigsten auftretende Mutation ist $\Delta \mathrm{F} 508$, die bei Nordeuropäern auf mindestens $70 \%$ der Chromosomen von CF-Patienten festgestellt wird. Bei den Südeuropäern ist diese Mutation sehr viel seltener. Beispiele für andere Mutationen, deren Prävalenz in den meisten Populationen ungefähr 1-2\% beträgt, sind u.a.: G542X, G551D, R553X, W1282X und N1303K. Zu guter Letzt gibt es ethnische Mutationen, die hauptsächlich in spezifischen Bevölkerungsgruppen vorkommen und dort eine Prävalenz von $1 \%$ bis sogar 7\% erreichen [25]. In den meisten Populationen machen diese weit verbreiteten Mutationen 80 bis 95\% aller mutanten CFTR-Gene aus.

Es gibt handelsübliche Assays, mittels derer das CFTRGen auf ungefähr 30 verschiedene Mutationen untersucht werden kann, die eine Prävalenz von ca. $0,5 \%$ haben und von denen die meisten mit der klassischen CF zusammenhängen [26]. Eine Mutationsdetektionsrate von $90 \%$ in einer bestimmten Population bedeutet, dass eine Mutation auf beiden CFTR-Genen bei $81 \%$ der typischen CF-Patienten identifiziert wird; eine Mutation auf nur 1 CFTR-Gen liegt bei $18 \%$ der Patienten vor und nur bei $1 \%$ der Patienten liegt keine CFTR-Mutation vor.

Wird bei einem Patienten mit Symptomen, die mit der atypischen zystischen Fibrose kompatibel sind, im Schweisstest ein grenzwertiges Ergebnis erzielt, so müssen für eine endgültige Diagnose möglicherweise beide
CFTR-Gene umfassend auf Mutationen untersucht werden. $100 \%$ Sensitivität wird nur durch Gen-Sequenzierung erzielt. Selbst wenn eine Mutation festgestellt wird, ist die Rolle, die diese Mutation bei der Erkrankung spielt, möglicherweise noch nicht klar. Bei vielen CFTR-Mutationen weiss man nicht, wie sich die Mutation auf die Genfunktion auswirkt; möglicherweise könnte es sich um Polymorphismen handeln [24].

\section{CFTR-Bioassays: Nasale Potenzialdifferenz (PD)}

Bei der CF handelt es sich um eine Erkrankung, die durch das Fehlen oder die Störung des CFTR-Proteins ausgelöst wird, das auf der apikalen Seite für die cAMPinduzierte Chloridsekretion verantwortlich ist. Darüber hinaus findet kompensatorisch ein übermässiger Natriumeinstrom in die Epithelzellen statt $[2,27]$. Die CFTRBioassays messen die Ionenflüsse im Epithel bzw. das sich daraus ergebende Spannungspotenzial an der Schleimhautoberfläche [28]. Sie bieten also einen direkten Einblick in die Physiologie der Zelle und der Ionenkanäle und wurden zur Lösung diagnostischer Probleme bei atypischen Patienten entwickelt, da mit Hilfe dieser Assays Störungen, die mit dem CFTR-Gen verbunden sind, bestätigt oder ausgeschlossen werden können. Diese Tests können am respiratorischen oder intestinalen Epithel durchgeführt werden, sind komplex und erfordern spezielle Geräte und Fachkenntnisse. Der Einsatz der nasalen Potenzialdifferenz (PD) als Test im Rahmen der CF-Diagnose wurde von Rosenstein und Cutting bereits 1998 in ihrem Konsenspapier zur Diagnose akzeptiert [29]. Gemessen wird die PD zwischen der Nasenschleimhaut und der Unterarmhaut. Einzelheiten zur praktischen Testdurchführung wurden bereits veröffentlicht $[28,30]$. Die vor Beginn des Tests gemessene Potenzialdifferenz gibt Hinweise auf den Natriumtransport über den amiloridsensitiven Natriumkanal des Epithels. Danach werden die Auswirkungen einer Blockade des epithelialen Natriumkanals unter Amilorid (Superfusion) bewertet. Abschliessend wird zur Messung der Chloridpermeabilität an der apikalen Membran ein starkes chemisches Chloridgefälle hergestellt.

In vielen grossen CF-Zentren ist dieser Test inzwischen Teil der klinische Praxis, und diese CF-Zentren haben eigene Richtwerte für diesen Test zusammengestellt. Bei gut ausgebildetem Personal und sorgfältiger Beachtung der technischen Details ist eine Standardisierung der nasalen Potenzialdifferenzmessung möglich [31].

Die nasale PD eines Patienten mit typischer CF ist leicht von der PD einer gesunden Kontrollperson zu unterscheiden (Abb. 1). 
Abb. 1. Messung der nasalen Potenzialdifferenz (PD) bei einer gesunden Person (a) und einer Person mit typischer CF (b). Bei einer gesunden Person ist die PD bei Beginn des Tests negativ ( -20 bis $-30 \mathrm{mV}$ ), steigt nach Amilorid-Applikation mässig an und fällt nach Verabreichung einer schwach konzentrierten Chloridlösung und Isoproterenol eindeutig ab. Bei einem Patienten mit typischer CF ist die PD zur Baseline noch negativer, steigt nach Amilorid-Applikation stärker an und verändert sich nach Verabreichung einer schwach konzentrierten Chloridlösung und Isoproterenol nicht. Aus DeBoeck et al. [55] mit freundlicher Genehmigung der BMJ Publishing Group.
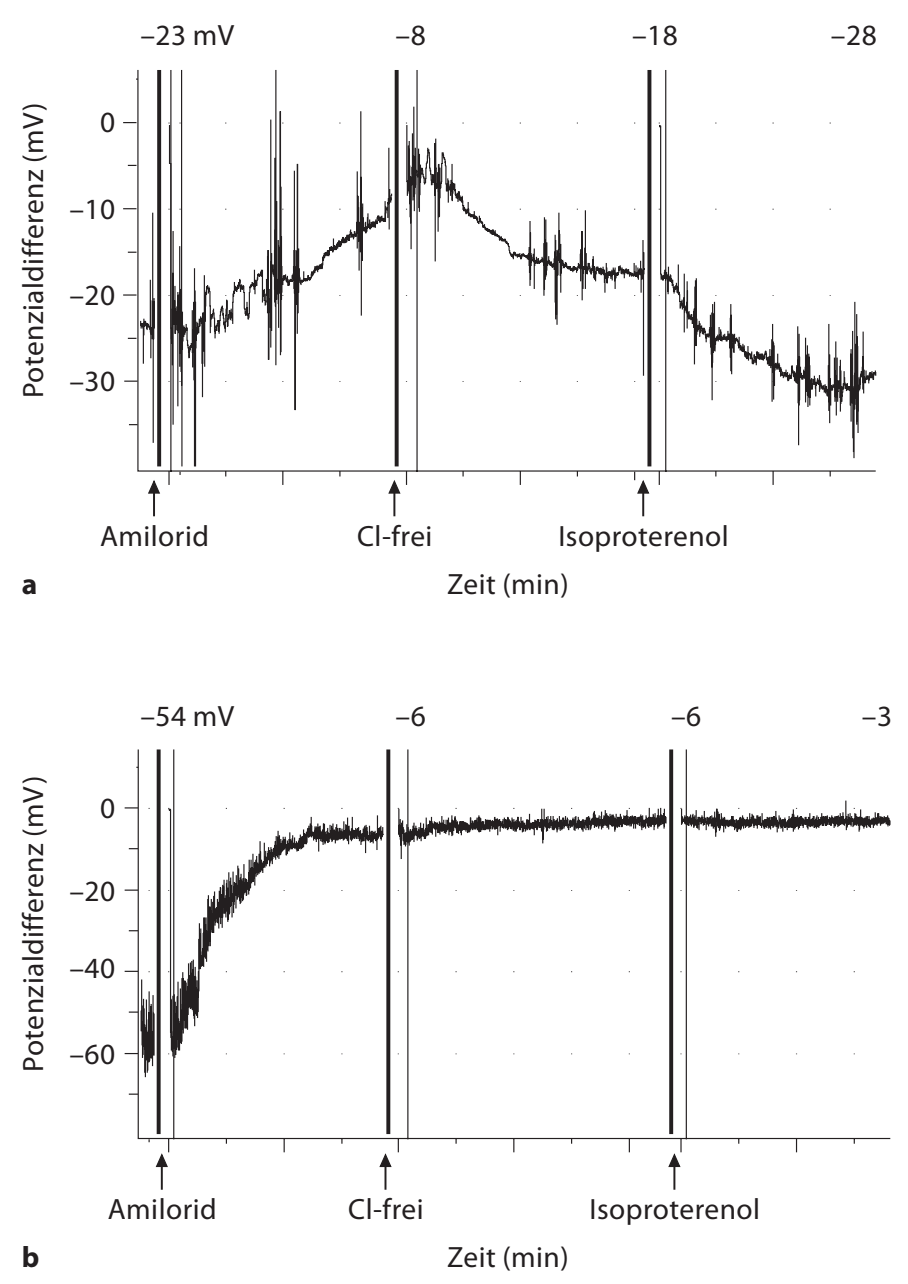

Bei einem CF-Patienten ist die vor Beginn des Tests gemessene Potenzialdifferenz negativer, die Amiloridreaktion ausgeprägter und die Reaktion auf die chloridfreie Lösung bzw. die Isoproterenol-Lösung schwächer oder nicht vorhanden. Bei Patienten mit atypischer CF kann das Ergebnis der nasalen Potenzialdifferenzmessung grenzwertig sein. Ein Konsens, ab wann ein Ergebnis als nicht mehr normal gilt, wurde bisher nicht erreicht.

\section{Klinische Aspekte}

Wie bereits erwähnt handelt es sich bei der CF um eine Multiorganerkrankung. $\mathrm{Zu}$ den charakteristischen CFSymptomen zählen chronische sinopulmonale Infekti- onen, gastrointestinale Störungen und Ernährungsstörungen, Salzverlustsyndrome und adrenogenitale Störungen, die zu einer obstruktiven Azoospermie führen (Tabelle 1). Bei fast allen Patienten tritt über kurz oder lang eine chronische sinopulmonale Erkrankung auf. Eine Pankreasinsuffizienz liegt bei ungefähr 90\% der Patienten vor. Männliche Patienten nach der Pubertät sind zeugungsunfähig.

\section{Erkrankungen der unteren Atemwege}

Die mit sporadischen Exazerbationen einhergehenden chronischen Infekte und Entzündungen der Lunge sind das Hauptproblem bei der CF und wirken sich bei den meisten CF-Patienten einschränkend auf die Lebensdauer und die Lebensqualität aus. Die Patienten leiden be- 
Tabelle 2. Faktoren, die sich bekannterweise auf den Krankheitsverlauf auswirken

\begin{tabular}{l} 
Nicht beeinflussbare Faktoren \\
Genotyp \\
Geschlecht \\
Status der Bauchspeicheldrüse \\
Polymorphismus und Gene, die auf Krankheiten \\
modifizierend wirken, z.B. TGF- $\beta$, Mannose-bindende \\
Lektine \\
\hline Beeinflussbare Faktoren \\
Alter bei Diagnose \\
Chronische Kolonisation mit P. aeruginosa oder B. cepacia \\
Schlechter Ernährungszustand \\
Schlechte körperliche Verfassung \\
Versorgung seitens des CF-Centers \\
Behandlungsintensität und Nachsorge \\
Exposition gegenüber Tabakrauch \\
Compliance
\end{tabular}

reits in einem frühen Lebensalter unter bakteriellen Infekten der Lunge, die bei ihnen mit einer verstärkten Entzündungsreaktion verbunden sind [32]. Aufgrund des gestörten Salz- und Wasserhaushalts in den Atemwegen kommt es dort zur Retention zäher Sekrete und zu einer Blockade. Dies trägt wesentlich zu den endlosen Infektionen und Entzündungen bei $[2,27]$. Der genaue Zusammenhang zwischen der CFTR-Mutation im Einzelnen und den chronischen bakteriellen Infekten ist jedoch noch nicht endgültig geklärt worden [Donaldson und Boucher, S. 103-109]. Zu den am häufigsten mit einer Lungeninfektion bei CF-Patienten in Verbindung stehenden Bakterien zählen Staphylococcus aureus, Haemophilus influenzae und Pseudomonas aeruginosa. Die Lungenerkrankung verläuft unweigerlich progressiv, wobei die Progressionsrate von vielen verschiedenen Faktoren abhängt (Tabelle 2). Der Ausbruch einer chronischen Pseudomonas-Infektion geht in der Regel mit einer rascheren Abnahme der Lungenfunktion einher [33]. Bei Patienten, bei denen die Funktion der Bauspeicheldrüse erhalten ist, nimmt die Lungenfunktion weniger stark ab [34]. Genotyp, modifizierend wirkende Gene, Geschlecht, Exposition gegenüber Tabakrauch, ein schlechter Ernährungsstatus, die Behandlungsintensität sowie weitere Faktoren wirken sich auf die Progression der Erkrankung aus [Cutting, S. 112-116].

Kennzeichnend für den klinischen Verlauf sind Episoden mit akuten Verschlechterungen der Lungeninfektion. Häufigstes und oft unterschätztes erstes Anzeichen einer Exazerbation ist ein verstärkter Husten. $\mathrm{Zu}$ den weiteren möglichen Symptomen und Anzeichen zählen Appetitlosigkeit und eine schlechtere Verträglichkeit von Sport und Bewegung, neue Auskultationsgeräusche, frische Infiltrate bei Röntgenaufnahmen der Brust und eine Abnahme der Lungenfunktion [35]. Die Patienten sind meist fieberfrei und weisen nicht alle der oben genannten Symptome auf. Die Exazerbationen treten tendenziell nach Virusinfektionen auf [36]. Je weiter die Krankheit fortschreitet, desto stärker ist die Zunahme von Husten und Sputumproduktion und die Einschränkung der Fähigkeit, Sport zu treiben und sich körperlich zu betätigen.

Die Ergebnisse der Lungenfunktionstests sind anfänglich normal, deuten jedoch bald auf eine Obstruktion der peripheren Atemwege hin. Der $\mathrm{FEV}_{1}$-Wert (forciertes expiratorisches Volumen in 1 Sekunde) ist die am häufigsten zur Beschreibung des Krankheitsfortschritts verwendete Variable [37]. Letztendlich kommt es zu der typischen Mischung aus Obstruktion/Restriktion und Hypoxie.

Das erste auf einer Röntgenaufnahme der Brust sichtbare pathologische Anzeichen ist die Überblähung, auf die bald Schleimretention, typischerweise im rechten oberen Lungenlappen, folgt. Diese Anzeichen verstärken sich bis hin zu breitstreifigen Verdichtungen, bronchialer Dilatation, Infiltraten, Zysten und Cor pulmonale. Quantifiziert werden diese Veränderungen anhand von ScoreSystemen [38].

Bei vielen Patienten liegt eine bestimmte Hyperreaktivität der Atemwege vor. Viele Patienten weisen ausserdem eine allergische Sensibilisierung auf das AspergillusAntigen auf, aber das vollständig ausgeprägte Syndrom der allergischen bronchopulmonalen Aspergillose tritt bei Progression der Erkrankung nur bei 10\% der Patienten auf [39]. Komplikationen im fortgeschrittenen Krankheitsstadium sind schwache und starke Hämoptyse und Pneumothorax. Ohne Lungentransplantation kommt es letztendlich zu Atemversagen und Tod.

\section{Erkrankungen der oberen Atemwege}

Bei fast allen Patienten sind auf den Röntgenaufnahmen Anzeichen einer Pansinusitis zu sehen, eine sich symptomatisch äussernde Sinusitis tritt jedoch selten auf. Viele Patienten leiden unter Nasenpolypen, die zu einer als störend empfundenen verstopften Nase führen können und unter Umständen entfernt werden müssen [40].

\section{Bauchspeicheldrüse}

Pankreasinsuffizienz ist bei CF-Patienten die Hauptursache für Gedeihstörungen. Sie tritt bei ungefähr $90 \%$ 
der Patienten für gewöhnlich im ersten Lebensjahr auf [7]. Zu den bei unbehandelten Patienten typischen Symptomen zählen lockere, fette Stühle, Flatulenz, Bauchschmerzen, exzessives Schreien und gesteigerter Appetit. Vorherrschendes Symptom ist die Fettmalabsorption. Mangelnde Compliance bei der Einnahme der Bauchspeichelenzyme stellt bei CF-Patienten nach wie vor die Hauptursache für Bauchschmerzen dar. Einige Genotypen werden mit einer besseren Erhaltung der Bauchspeicheldrüsenfunktion in Verbindung gebracht. Bei diesen Patienten liegt eine höhere Anfälligkeit für Episoden der akuten Pankreatitis in der Jugend und im Erwachsenenalter vor [41]. Die relative Glukose-Intoleranz nimmt mit dem Alter zu und die Prävalenz des mit der CF in Zusammenhang stehenden Diabetes mellitus beträgt bei jungen Erwachsenen über 20\%. Diabetes mellitus wird vor und nach der Diagnose mit einem schlimmeren Krankheitsverlauf in Verbindung gebracht [42].

\section{Darm}

Bei ungefähr 15\% der Neugeborenen mit CF kommt es, weil die Darmpassage im distalen Dünndarm mit zähem Mekonium blockiert ist, zu einem Mekonium-Ileus [4]. Alle CF-Patienten sind auch nach Abschluss der Neugeborenenphase anfällig für ähnliche Episoden der Dünndarmobstruktion, die auch als distales intestinales Obstruktionssyndrom (DIOS) bezeichnet wird [43]. Die ersten Zeichen sind krampfartige Bauchschmerzen und eine herabgesetzte Stuhlproduktion. Werden diese Zeichen nicht rechtzeitig erkannt, so können sie einen kompletten Darmverschluss mit abdomineller Distension und Erbrechen zur Folge haben. Blinddarmentzündungen verlaufen bei CF-Patienten atypisch und können nach stummer Perforation zu einem Abszess im rechten unteren Quadranten des Abdomens führen [44]. Häufiger kommt es $\mathrm{zu}$ einer Intussuszeption. Bei einigen Säuglingen mit CF kommt es hauptsächlich vor Einsetzen der Behandlung rezidivierend zu einem mit Malabsorption einhergehenden Prolaps.

\section{Leber-Gallen-Trakt}

Die Cholestase im Neugeborenenalter kann zur Diagnose CF führen, wobei jedoch kein Zusammenhang mit einer im späteren Leben auftretenden Lebererkrankung besteht [5]. Bei vielen Patienten tritt eine asymptomatische Erhöhung der Leberenzyme auf. Einige wenige entwickeln eine schwerwiegende biliäre Zirrhose, die eine Lebertransplantation erforderlich macht, oder sterben an den Komplikationen der portalen Hypertension und Splenomegalie [45]. Klinische Anzeichen, wie z.B. eine vergrösserte Milz und eine tastbar vergrösserte Leber mit einer harten Kante lassen auf eine Lebererkrankung schliessen. Die zuverlässigsten Informationen gewinnt man durch eine Leberbiopsie, die aber, da es sich um einen invasiven Eingriff handelt, nicht häufig praktiziert wird. Die meisten Zentren führen jährliche eine Ultraschalluntersuchung der Leber durch, um den von Westaby definierten Ultraschall-Score festzustellen und Patienten mit signifikanten Lebererkrankungen so frühzeitig zu erkennen.

\section{Ösophagus}

Säuglinge mit CF leiden häufig unter gastroösophagealem Reflux [47], der sich durch die Physiotherapie, bei der der Kopf nach unten geneigt wird, noch verschlimmern kann. Auch bei älteren CF-Patienten tritt häufig gastroösophagealer Reflux auf. Zu den Faktoren, die einen gastroösophagealen Reflux verstärken können, zählen Überblähung, Husten und erhöhte Anstrengungen beim Einatmen.

\section{Ernährungszustand}

Mit fortschreitender Erkrankung verschlechtert sich tendenziell auch der Ernährungsstatus der CF-Patienten, bei denen möglicherweise Wachstumshemmung, ein geringeres Körpergewicht und eine verzögerte sexuelle Reifung vorliegen können. Um ein normales Längenwachstum und ein normales Körpergewicht zu erreichen, bedarf es grosser Anstrengungen. Die CF-Patienten leiden unter Malabsorption, sie haben, da sie ihre chronischen Infektionen bekämpfen müssen, einen höheren Kalorienbedarf und möglicherweise eine an sich erhöhte Stoffwechselrate [48]. Sie sind stark anfällig für einen Mangel an fettlöslichen Vitaminen, Spurenelementen und essenziellen Fettsäuren [49] und leiden unter einem gestörten Fettsäurestoffwechsel [50; Strandvik, S. 132].

\section{Weitere Symptome}

Die sexuelle Reifung ist bei relativ vielen CF-Patienten verzögert. Männliche CF-Patienten sind nach der Pubertät fast immer zeugungsunfähig. Bei Frauen ist die Fruchtbarkeit herabgesetzt und eine Schwangerschaft kann bei verminderter Lungenfunktion gesundheitsbedrohlich sein [51].

Ein durch Schwitzen verursachter übermässiger Salzverlust kann eine akute Dehydratation zur Folge haben [9]. Bei Säuglingen mit CF kann eine zu geringe Salzaufnahme in Kombination mit einem übermässigen Salzverlust an heissen Tagen zur metabolischen Alkalose führen [9]. 
Das Cor pulmonale ist eine Folge der fortgeschrittenen Lungenerkrankung und anhaltenden Hypoxie.

Bei den meisten Patienten mit fortgeschrittener Lungen- oder Lebererkrankung werden Trommelschlegelfinger beobachtet. Bei jungen Erwachsenen tritt Osteoporose auf [52]. Da chronische Infektionen inzwischen intensiver behandelt werden, ist die typische hypertrophe Osteoarthropathie selten geworden.

\section{Psychische Probleme}

Immer mehr CF-Patienten führen trotz ihrer Erkrankung und der belastenden Behandlung ein aktives und produktives Leben. Trotzdem sind viele von ihnen aufgrund ihrer schwachen Gesundheit und ungewissen $\mathrm{Zu}$ kunft dem Risiko emotionaler und psychischer Probleme ausgesetzt. Eine Arbeitsstelle und einen Lebenspartner $\mathrm{zu}$ finden bzw. am normalen Leben teilzunehmen, ist nicht einfach, wenn man an einer lebensverkürzenden Erkrankung leidet. Die Zahl der Komplikationen wächst mit zunehmendem Alter und die Behandlung wird immer komplexer und teurer. Häufig jedoch entspricht die von den Patienten wahrgenommene psychische Belastung nicht ihrem tatsächlichen objektiven Gesundheitsstatus, d.h. Depressionen können durchaus bei Patienten auftreten, die laut Auskunft ihres Arztes keine nennenswerten Gesundheitsprobleme haben [53, 54].

Auch bei den Eltern und Geschwistern von CF-Patienten ist das Risiko für psychische Probleme aufgrund des emotionalen Stresses, der Einweisungen ins Krankenhaus, der finanziellen Belastungen, der für die Pflege des kranken Kindes benötigten Zeit und des Gefühls des Ausgegrenztseins erhöht.

\section{Atypische CF}

Man weiss bereits seit langem, dass es CF-Patienten gibt, bei denen die Funktion der Bauchspeicheldrüse erhalten bleibt, was auf ein variables Krankheitsspektrum schliessen lässt. Seit Entdeckung des CFTR-Gens ist zunehmend klar, dass die typischen, in einer frühen Lebensphase einsetzenden und mit der klassischen CF verbundenen CF-Symptome nur einen kleinen Teil der möglicherweise mit einer CFTR-Störung einhergehenden Symptome darstellen. Es gibt Patienten, bei denen die Symptome schwach ausgeprägt sind und die Erkrankung erst in der Jugend oder dem Erwachsenenalter auftritt, und Patienten, die nur ein einziges klinisches Merkmal aufweisen, z.B eine rezidivierende Pankreatitis, eine sklerosierende Cholangitis, eine idiopathische Bronchiektasie oder Zeugungsunfähigkeit. Bei den beiden letzten Kategorien spricht man von einer atypischen CF. Bei den meisten dieser Patienten wird im Schweisstest ein grenzwertiger Chloridwert, d.h. ein Chloridwert zwischen 30 und $60 \mathrm{mEq} / \mathrm{l}$, ermittelt. In der von Rosenstein und Cutting [29] verfassten amerikanische Konsenserklärung zur Diagnose von CF wurden die Diagnosekriterien anhand dieser Erkenntnisse neu definiert. Laut diesen beiden Wissenschaftlern bleibt die Diagnose CF bestehen, wenn ein Patient mindestens eines der phänotypischen Merkmale aufweist oder ein Geschwister unter CF leidet oder in einer neonatalen Untersuchung ein positives Ergebnis erzielt und zudem die CFTR-Mutation im Labor nachgewiesen wurde, und zwar entweder durch ein eindeutig positives Ergebnis des Schweisstests oder zwei krankheitsursächliche CFTR-Mutationen oder ein pathologisches Ergebnis der nasalen Potenzialdifferenzmessung [29]. Diese Definition unterscheidet jedoch nicht zwischen Patienten mit klassischer CF und Patienten mit weniger stark ausgeprägten Phänotypen. Die Autoren einer europäischen Konsenserklärung jüngeren Datums waren pragmatischer: Die Bezeichnung typische CF bleibt Patienten mit klinischen Symptomen und einem positiven Schweisstest vorbehalten, wohingegen die Bezeichnung atypische CF Patienten mit zweifelhaftem oder normalem Ergebnis des Schweisstests vorbehalten bleibt, bei denen die Diagnose durch moderne diagnostische Werkzeuge gestützt wird, d.h. zwei krankheitsursächliche Mutationen oder ein pathologisches Ergebnis der nasalen Potenzialdifferenzmessung [55]. Diese Klassifizierung erweist sich als nützlich, ihre Anwendung jedoch sollte nicht zu rigide sein. Der CF-Phänotyp stellt in der Tat ein Kontinuum dar, bei dem sich die einzelnen Kategorien überschneiden können.

Die Diagnose CF, die für eine lebensverkürzende Krankheit steht, bedeutet eine grosse emotionale Bürde und bringt für Patienten möglicherweise Nachteile mit sich, z.B. im Hinblick auf Arbeits- oder Versicherungsverhältnisse. Aus diesem Grund sollte bei Patienten mit atypischer CF nachdrücklich betont werden, dass sie nicht an der typischen CF leiden. Noch besser wäre es, Patienten, bei denen die Krankheit sich in nur einem Organ manifestiert, nach den Symptomen zu klassifizieren und von der Diagnose atypische CF Abstand zu nehmen, z.B. Zeugungsunfähigkeit aufgrund des Fehlens beider Samenleiter, sklerosierende Cholangitis oder eine andere, im Diagnoseschlüssel der WHO enthaltene Bezeichnung [56]. Diese Patienten müssen aus zwei Gründen beobachtet werden: Erstens können sich die klinischen Merkmale bei den einzelnen Patienten im Laufe der Zeit verändern, d.h. ein Patient, bei dem lediglich die Samenleiter auf beiden Seiten fehlen, entwickelt möglicherweise später eine 
Lungeninfektion, und zweitens müssen sich die Ärzte öffnen und ihr Wissen darüber, was eine CFTR-Störung für das Leben eines Patienten bedeuten kann, mit anderen teilen.

Patienten mit einem unbestimmten Chloridgehalt im Schweisstest, bei denen nur eine CFTR-Mutation nachgewiesen wurde und bei denen die nasale Potenzialdifferenzmessung kein eindeutiges Ergebnis bringt, zählen zu den Fällen, bei denen eine Diagnose eine echte Herausforderung darstellt: Eine eindeutige Klassifikation dieser Patienten ist derzeit nicht möglich, weil eine durch die CFTR-Mutation verursachte Störung nicht eindeutig nachgewiesen werden kann. Sie sind jedoch zumindest CF-Träger. Bei persistierenden Symptomen bedarf es einer strukturierten Nachsorge in einer geeigneten Einrichtung und einer symptomatischen Behandlung. Eine genetische Beratung ist für diese Patienten und Ihre Familien wichtig. Die Längsschnittdaten dieser Patienten müssen erfasst werden, um so das Wissen und die Einblicke in diese Patientengruppe zu erhöhen: Eine Möglichkeit ist eine Kombination aus einer Mutation und mehreren ungünstigen Modifier-Genen.

\section{Beratung}

Da es sich bei der Beratung von Familien und Verwandten um eine höchst komplexe, emotionsgeladene Aufgabe handelt, die darüber hinaus das Risiko birgt, dass richtige Informationen möglicherweise fehlgedeutet, überinterpretiert oder nur unvollständig im Gedächtnis behalten werden, sollte eine solche Beratung von einem klinischen Genetiker durchgeführt werden. Die Beratung der Familie von der Pflege des Patienten abzukoppeln, erweist sich ebenfalls als Vorteil.

\section{Eltern von Kindern mit CF}

Die Eltern von Kindern, die an CF leiden, sind zwangsläufig heterozygot. Bei diesen Eltern besteht eine Wahrscheinlichkeit von eins zu vier, dass jedes weitere Kind ebenfalls an CF erkrankt. Betroffene Eltern, die sich weitere Kinder wünschen, sollten die Möglichkeit haben, eine pränatale Untersuchung durchführen zu lassen. Sind beide Mutationen bekannt, wird an mittels Chorionzottenbiopsie oder Amniozentese gewonnenen fetalen Zellen eine Mutationsanalyse durchgeführt [57]. Wurde lediglich eine krankheitsursächliche CFTR-Mutation nachgewiesen, so muss keine weitere DNA-Untersuchung durchgeführt werden. Eine Pränataldiagnose ist auch mittels Segregationsanalyse von Polymorphismen mög- lich, die innerhalb des CFTR-Gens auftreten oder mit diesem verbunden sind. Aufgrund der mit einem Schwangerschaftsabbruch einhergehenden psychischen Belastung entscheiden sich viele Eltern gegen weitere Schwangerschaften $[58,59]$.

\section{Geschwister von CF Patienten}

Selbst innerhalb einer Familie kann sich die CF äusserst unterschiedlich manifestieren. Daher sollte bei Geschwistern von Kindern, die unter CF leiden, eine mögliche CF-Erkrankung nicht aufgrund von klinischen Symptomen, sondern mittels formeller Tests ausgeschlossen werden. Bei kleinen Kindern wird geraten, eine CFErkrankung eher mittels Schweisstest als mittels Mutationsanalyse auszuschliessen, da die Frage, ob Minderjährige Träger einer CF-Mutation sind, unwichtig und nur bei Geschwisterkindern im Rahmen von Reproduktionsentscheidungen von Bedeutung ist. Es besteht die Sorge, dass Kinder, die eine CF-Mutation in sich tragen, von ihren Eltern übermässig behütet oder auf andere Weise stigmatisiert werden könnten [60].

\section{Angehörige der Eltern von Kindern mit CF}

Auch die Verwandten von Eltern, deren Kinder unter CF leiden, haben ein höheres Risiko, Träger einer CFMutation zu sein, und sollten darüber informiert werden. Einige Genetiker raten, eher Paare als Einzelpersonen über ihren Risikostatus zu informieren [61], angesichts der heutigen Zeit, in der Sexualpartner häufig wechseln, erweist sich dies jedoch als eine komplexe Aufgabe. Wird eine Person nicht als Träger einer CF-Mutation identifiziert, so verringert sich zwar das Risiko, ein CF-krankes Kind zu bekommen, kann aber niemals ganz ausgeschlossen werden. Screeningtests berücksichtigen jeweils nur die Mutationen mit der höchsten Prävalenz und die bei dem CF-Patienten exprimierten Mutationen, vorausgesetzt diese waren nicht in dem üblichen Umfang der Untersuchung enthalten.

\section{Schlussfolgerungen}

Bei der CF handelt es sich um eine komplexe, durch eine Störung des CFTR-Proteins verursachte Multiorganerkrankung.

Bei den mit Abstand meisten Fällen geht die CF mit einem vollständigen Verlust der CFTR-Funktion einher und die Diagnose CF ist eindeutig: Das klinische Bild ist klar und die klinische Diagnose wird von den im Schweisstest erzielten Ergebnissen bestätigt. Normaler- 
weise wird in beiden CFTR-Genen eine typische krankheitsursächliche CFTR-Mutation entdeckt. Bei einer geringen Zahl an Patienten ist das klinische Bild viel schwächer ausgeprägt oder sogar auf ein Organ begrenzt. Hierbei handelt es sich um die Gruppe der atypischen CFPatienten. Bei den meisten dieser Patienten wird die Diagnose durch eine CFTR-Mutationsanalyse oder Bioassays der CFTR-Proteinfunktion bekräftigt. Bei seltenen CFTRMutationen kann manchmal nicht belegt werden, dass diese bei atypischen CF-Fällen tatsächlich krankheitsursächlich sind. Über die langfristige Prognose dieser atypischen Fälle wissen wir bisher noch zu wenig. Diese Patienten leiden nicht unter einer typischen CF und sollten dies auch eindeutig so vermittelt bekommen.

Die erste Manifestation der CF kann mittels vieler verschiedener Symptome erfolgen, am häufigsten jedoch äussert sich die Erkrankung durch chronischen Husten und lockere Stühle im Kindesalter. Insbesondere bei weniger stark ausgeprägten Fällen ist ein hoher klinischer Verdachtsindex das beste Mittel, um die CF-Diagnose nicht $\mathrm{zu}$ verfehlen. Der Pilocarpin-IontophoreseSchweisstest ist nach wie vor der Test der Wahl. Die Chloridkonzentration im Schweiss beträgt bei CF-Patienten $>60 \mathrm{mmol} / \mathrm{l}$. Der Test ist zwar einfach, sollte aber aus Gründen der klinischen Genauigkeit in einem Speziallabor durchgeführt werden. In schwierigen Fällen erweisen sich eine CFTR-Analyse und CFTR-Bioassays zusätzlich als hilfreich. Eine frühzeitige Diagnose ist notwendig, um die Progression der Erkrankung zu verzögern.

Chronische, progressive bakterielle sinopulmonale Infektionen und Entzündungen mit zeitweiligen Exazerbationen sind die typischsten Manifestationen der Erkrankung. Neunzig Prozent der Patienten leiden unter einer Insuffizienz der Bauchspeicheldrüse. Männliche Patienten sind nach der Pubertät zeugungsunfähig. Bei CF-Patienten besteht lebenslang ein Risiko für Krankheitsmanifestationen und Komplikationen in diversen Organen: den Nasennebenhöhlen, der Lunge, der Bauchspeicheldrüse, der Leber, dem Darm, dem Ösophagus, den Knochen, den Gelenken etc. Die komplexe Mischung aus möglichen Krankheitsmanifestationen ist bei jedem Patienten einzigartig. Mit zunehmendem Alter nimmt die Zahl der Komplikationen tendenziell zu: So leiden beispielsweise 20\% der jungen Erwachsenen mit CF an einem damit verbundenen Diabetes mellitus.

Die klassische CF ist eine lebensverkürzende Erkrankung und die mittlere Lebensdauer beträgt selbst bei intensivster Behandlung und Beobachtung derzeit ungefähr 34 Jahre. Dies stellt für die Patienten und ihre Familien eine enorme psychische Belastung dar.

Beratungsleistungen, die darauf abzielen, weitere Erkrankungen bei Geschwistern und im weiteren Familienkreis zu verhindern, sind wichtig, aber komplex.

\section{Literatur}

1 Report of a Joint Meeting of WHO/ECFTN/ ICF/(M)A/ECFS: The Molecular Genetic Epidemiology of Cystic Fibrosis. Geneva, WHO, 2006. http//:www.who.int/genomics/ publications/en/

-2 Boucher RC: Regulation of airway surface liquid volume by human airway epithelia. Pflugers Arch 2003;445:495-498.

3 Rosenstein BJ: Diagnostic methods; in Hodson M, Geddes D (eds): Cystic Fibrosis. London, Arnold, 2006, vol 2, pp 177-188.

4 Mushtaq I, Wright VM, Drake DP, et al: Meconium ileus secondary to cystic fibrosis. The East London experience. Pediatr Surg Int 1998;13:365-369.

5 Shapira R, Hadzic N, Francavilla R, et al: Retrospective review of cystic fibrosis presenting as infantile liver disease. Arch Dis Child 1999;81:125-128.

6 Lai HJ, Cheng Y, Farrell PM: The survival advantage of patients with cystic fibrosis diagnosed through neonatal screening: evidence from the United States Cystic Fibrosis Foundation registry data. J Pediatr 2005; 147 : S57-S63.
7 Waters DL, Dorney SF, Gaskin KJ, et al: Pancreatic function in infants identified as having cystic fibrosis in a neonatal screening program. N Engl J Med 1990;322:303-308.

-8 Abman SH, Accurso FJ, Butler-Simon N, et al: Role of respiratory syncytial virus in early hospitalizations for respiratory distress of young infants with cystic fibrosis. J Pediatr 1988; 113:826-830.

-9 Sojo A, Rodriguez-Soriano J, Vitoria JC, et al: Chloride deficiency as a presentation or complication of cystic fibrosis. Eur J Pediatr 1994;153:825-828.

10 Chillon M, Casals T, Mercier B, et al: Mutations in the cystic fibrosis gene in patients with congenital absence of the vas deferens. N Engl J Med 1995;332:1475-1480.

11 Muniz AE, Bartle S, Foster R: Edema, anemia, hypoproteinemia, and acrodermatitis enteropathica: an uncommon initial presentation of cystic fibrosis. Pediatr Emerg Care 2004;20:112-114.
12 Grosse SD, Boyle CA, Botkin JR, et al: Newborn screening for cystic fibrosis: evaluation of benefits and risks and recommendations for state newborn screening programs. MMWR Recomm Rep 2004;53:1-36.

13 Scotet V, de Braekeleer M, Roussey M, et al: Neonatal screening for cystic fibrosis in Brittany, France: assessment of 10 years' experience and impact on prenatal diagnosis. Lancet 2000;356:789-794.

14 Gibson LE, Cooke RE: A test for concentration of electrolytes in sweat in cystic fibrosis of the pancreas utilizing pilocarpine by iontophoresis. Pediatrics 1959;23:545-549.

15 Orenstein DM, Winnie GB, Altman H: Cystic fibrosis: a 2002 update. J Pediatr 2002; 140:156-164

16 LeGrys VA: Sweat testing for the diagnosis of cystic fibrosis: practical considerations. J Pediatr 1996;129:892-897.

17 Guidelines for the performance of the sweat test for the investigation of cystic fibrosis in the UK. Report from the multi-disciplinary working group. July 2002 (http//:www.acb. org.uk). 
-18 Di Sant'Agnese PA, Darling RC, Perera GA, Shea E: Abnormal electrolyte composition of sweat in cystic fibrosis of the pancreas; clinical significance and relationship to the disease. Pediatrics 1953;12:549-563.

19 Kirk JM, Keston M, McIntosh I, Essa S: Variation of sweat sodium and chloride with age in cystic fibrosis and normal populations: further investigations in equivocal cases. Ann Clin Biochem 1992;29:145-152.

20 Lebecque P, Leal T, De Boeck C, et al: Mutations of the cystic fibrosis gene and intermediate sweat chloride levels in children. Am J Respir Crit Care Med 2002;165:757-761.

21 National Committee for Clinical Laboratory Standards (NCCLS): Sweat Testing: Sample Collection and Quantitative Analysis. Approved guideline C34-A2. Wayne, NCCLS, 2006.

-22 Highsmith WE, Burch LH, Zhou Z, et al: A novel mutation in the cystic fibrosis gene in patients with pulmonary disease but normal sweat chloride concentrations. N Engl J Med 1994;331:974-980.

23 Cystic Fibrosis Genotype-Phenotype Consortium: Correlation between genotype and phenotype in patients with cystic fibrosis. N Engl J Med 1993;329:1308-1313.

24 http://genet.sickkids.on.ca/cgi-bin/WebObjects/MUTATION

-25 Schaedel C, Hjelte L, de Monestrol I, et al: Three common CFTR mutations should be included in a neonatal screening programme for cystic fibrosis in Sweden. Clin Genet 1999;56:318-322.

26 Dequeker E, Cuppens H, Dodge J, et al: Recommendations for quality improvement in genetic testing for cystic fibrosis. European Concerted Action on Cystic Fibrosis. Eur J Hum Genet 2000;8(suppl 2):S2-S24.

-27 Donaldson SH, Boucher RC: Update on pathogenesis of cystic fibrosis lung disease. Curr Opin Pulm Med 2003;9:486-491.

28 Knowles MR, Paradiso AM, Boucher RC: In vivo nasal potential difference: techniques and protocols for assessing efficacy of gene transfer in cystic fibrosis. Hum Gene Ther 1995;6:445-455.

29 Rosenstein BJ, Cutting GR: The diagnosis of cystic fibrosis: a consensus statement. Cystic Fibrosis Foundation Consensus Panel. J Pediatr 1998;132:589-595.

30 Schuler D, Sermet-Gaudelus I, Wilschanski $\mathrm{M}$, et al: Basic protocol for transepithelial nasal potential difference measurements. J Cyst Fibros 2004;3(suppl 2):151-155.
Standaert TA, Boitano L, Emerson J, et al: Standardized procedure for measurement of nasal potential difference: an outcome measure in multicenter cystic fibrosis clinical trials. Pediatr Pulmonol 2004;37:385-392.

32 Armstrong DS, Grimwood K, Carlin JB, et al: Lower airway inflammation in infants and young children with cystic fibrosis. Am J Respir Crit Care Med 1997;156:1197-1204.

33 Frederiksen B, Koch C, Hoiby N: Antibiotic treatment of initial colonization with $\mathrm{Pseu}$ domonas aeruginosa postpones chronic infection and prevents deterioration of pulmonary function in cystic fibrosis. Pediatr Pulmonol 1997;23:330-335.

34 Corey M, Edwards L, Levison H, Knowles M: Longitudinal analysis of pulmonary function decline in patients with cystic fibrosis. J Pediatr 1997;131:809-814.

35 Rosenfeld M, Emerson J, Williams-Warren J, et al: Defining a pulmonary exacerbation in cystic fibrosis. J Pediatr 2001;139:359-365.

36 Collinson J, Nicholson KG, Cancio E, et al: Effects of upper respiratory tract infections in patients with cystic fibrosis. Thorax 1996; 51:1115-1122.

37 Corey M, McLaughlin FJ, Williams M, Levison $\mathrm{H}$ : A comparison of survival, growth, and pulmonary function in patients with cystic fibrosis in Boston and Toronto. J Clin Epidemiol 1988;41:583-591.

38 Brasfield D, Hicks G, Soong S, Tiller RE: The chest roentgenogram in cystic fibrosis: a new scoring system. Pediatrics 1979;63:24-29.

$>39$ Mastella G, Rainisio M, Harms HK, et al: Allergic bronchopulmonary aspergillosis in cystic fibrosis. Eur Respir J 2001;17:10521053.

40 Jorissen MB, De Boeck K, Cuppens H: Genotype-phenotype correlations for the paranasal sinuses in cystic fibrosis. Am J Respir Crit Care Med 1999;159:1412-1416.

41 De Boeck K, Weren M, Proesmans M, Kerem E: Pancreatitis among patients with cystic fibrosis: correlation with pancreatic status and genotype. Pediatrics 2005;115:e463-e469.

42 Moran A, Hardin D, Rodman D, et al: Diagnosis, screening and management of cystic fibrosis related diabetes mellitus: a consensus conference report. Diabetes Res Clin Pract 1999;45:61-73.

43 Mascarenhas MR: Treatment of gastrointestinal problems in cystic fibrosis. Curr Treat Options Gastroenterol 2003;6:427-441.

44 Martens M, De Boeck K, Van Der Steen K, et al: A right lower quadrant mass in cystic fibrosis: a diagnostic challenge. Eur J Pediatr 1992;151:329-331.

45 Sokol RJ, Durie PR: Recommendations for management of liver and biliary tract disease in cystic fibrosis. Cystic Fibrosis Foundation Hepatobiliary Disease Consensus Group. J Pediatr Gastroenterol Nutr 1999;28(suppl 1): S1-S13.
46 Williams SG, Evanson JE, Barrett N, et al: An ultrasound scoring system for the diagnosis of liver disease in cystic fibrosis. J Hepatol 1995;22:513-521.

47 Heine RG, Button BM, Olinsky A, et al: Gastro-oesophageal reflux in infants under 6 months with cystic fibrosis. Arch Dis Child 1998;78:44-48.

48 Sinaasappel M, Stern M, Littlewood J, et al: Nutrition in patients with cystic fibrosis: a European Consensus. J Cyst Fibros 2002;1: 51-75.

49 Roulet M, Frascarolo P, Rappaz I, Pilet M: Essential fatty acid deficiency in well nourished young cystic fibrosis patients. Eur J Pediatr 1997;156:952-956.

50 Strandvik B, Gronowitz E, Enlund F, et al: Essential fatty acid deficiency in relation to genotype in patients with cystic fibrosis. J Pediatr 2001;139:650-655.

51 Edenborough FP, Stableforth DE, Webb AK, et al: Outcome of pregnancy in women with cystic fibrosis. Thorax 1995;50:170-174.

52 Conway S: Osteoporosis is cystic fibrosis. J Cyst Fibros 2003;2:161-162.

53 Elgudin L, Kishan S, Howe D: Depression in children and adolescents with cystic fibrosis: case studies. Int J Psychiatry Med 2004;34: 391-397.

54 Kotwicki RJ, Condra L, Vermeulen L, et al: Assessing the quality of life in children with cystic fibrosis. WMJ 2001;100:50-54.

55 De Boeck K, Wilschanski M, Castellani C, et al: Cystic fibrosis: terminology and diagnostic algorithms. Thorax 2005.

56 Classification of cystic fibrosis and related disorders. J Cyst Fibros 2002;1:5-8.

57 Richards CS, Grody WW: Prenatal screening for cystic fibrosis: past, present and future. Expert Rev Mol Diagn 2004;4:49-62.

58 Evers-Kiebooms G, Denayer L, Cassiman JJ, Van den Berghe H: Family planning decisions after the birth of a cystic fibrosis child: the impact of prenatal diagnosis. Scand J Gastroenterol 1988;23:38-46.

59 Mischler EH, Wilfond BS, Fost N, et al: Cystic fibrosis newborn screening: impact on reproductive behavior and implications for genetic counseling. Pediatrics 1998;102:4452.

60 Clarke A: The genetic testing of children Working Party of the Clinical Genetics Society (UK). J Med Genet 1994;31:785-797.

-61 Super M, Schwarz MJ, Malone G, et al: Active cascade testing for carriers of cystic fibrosis gene. BMJ 1994;308:1462-1467. 